Article available at http://wWw.parasite-journal.org or nitp://dx.dol.org/10.1051/parasite/199/044343

\title{
Aedes atropalpus, un nouveau moustique importé en Italie LORS DE TRANSPORTS DE PNEUS USAGÉS
}

\author{
ROMI R.*, SABATINELLI G.* \& PONTUALE G.*
}

Summary : AEDES ATROPALPUS, A NEW MOSQUITO INTRODUCED BY DISCARDED TIRES IN ITALY

In late September 1996, during a routine survey for the surveillance of Aedes albopictus in Italy, a breeding population of Aedes (Ochlerotatus) atropalpus (Coquillet, 1902) was discovered in Veneto Region, Italy. Ae. atropalpus is a typical rock hole neoartic mosquito which have been frequently collected in man made containers such as used or discarded tires. Since tire casing from United States was the primary mode of introduction of Ae. albopictus in Italy, a similar path could be taken by Ae. atropalpus. In the USA this species is an important daylightbiting pest mosquito and a potential vector of some arboviruses and avian plasmodia. The presence of the species in the same site was confirmed in late August 1997.

KEY WORDS : mosquitoes, Aedes atropalpus, Italy, discarded tires

MOTS CLÉS : moustiques, Aedes atropalpus, Italie, pneus usagés.

\begin{abstract}
Résumé :
Dans la seconde moitié du mois de septembre 1996, un nouveau moustique a été découvert en Italie: Aedes (Ochlerotatus) atropalpus (Coquillet, 1902). Un gîte larvaire de cette espèce a été trouvé dans la province de Trévise (Vénétie) par des opérateurs de l'Unité Sanitaire Locale lors d'opérations de routine menées dans le cadre du programme de surveillance d'Aedes albopictus en Vénétie. II s'agit d'un moustique néarctique dont les larves se développent habituellement dans des petites collections d'eau qui se forment dans les trous de rocher remplis d'eau, le long des torrents de montagne. Récemment, aux États-Unis, l'espèce s'est adaptée à différents types de récipients résultant des activités humaines, et en particulier aux pneus usagés entreposés en plein air. C'est ainsi que, comme pour Ae. albopictus, les œuts de cette nouvelle espèce ont été introduits en ltalie lors de l'importation de pneus usagés provenant des États-Unis. Ae. atropalpus étant un moustique à l'activité trophique principalement diurne, il peut être de ce fait une source de nuisance considérable; il est, en outre, un vecteur potentiel de certains arbovirus et plasmodium d'oiseaux. La présence de l'espèce dans le même lieu de récolte a été confirmé fin août 1997.
\end{abstract}

dans d'autres zones d'Italie (Romi, 1995; Knudsen et al., 1996).

Dans la seconde moitié du mois de septembre 1996, lors d'enquêtes de routine effectuées par les opérateurs de l'ASL de Trévise dans la localité de Villorba $(5 \mathrm{~km}$ au nord-est de Trévise) quelques exemplaires de larves et d'adultes d'un Aedes appartenant ou sous-genre Ochlerotatus ont été récoltés; elles ne pouvaient être rapportées à aucune des espèces de la faune italienne actuellement connues. Ces exemplaires ont ensuite été identifiés au Centre de Référence pour la Systématique des Culicidés de l'Istituto Superiore di Sanità de Rome comme appartenant à une espèce d'Amérique du Nord: Aedes atropalpus (Coquillet, 1902).

Les larves de cet Aedes ainsi que celles de Ae. albopictus ont été trouvées à l'intérieur de pneus usagés de grandes dimensions (camions, machines de chantiers), entreposés en plein air par deux petites entreprises qui rechapent les pneus usagés. Une enquête approfondie a permis d'établir qu'une des deux entreprises importe des pneus d'Amérique du Nord et, en particulier, des États-Unis. Dans les deux dernières années, les chargements sont parvenus au rythme de

\footnotetext{
* Istituto Superiore di Sanità, Laboratoire de Parasitologie, Viale Regina Elena 299, 00161 Rome, Italie.

Correspondence : Roberto Romi.

Tél. : ++39-6-49902301 - Fax. : ++39-6-49387065.

E-mail: romi@pop3.iss.it.
} 
deux containers par mois, contenant chacun 20 à 25 pneus usagés de grandes dimensions, en provenance du Texas et du Minnesota. Comme cela a déjà été démontré précédemment pour Ae. albopictus (Dalla Pozza et al., 1994), on peut supposer que les oufs d'Ae. atropalpus ont été introduits en Italie lors de ces transports.

Suite à la découverte d'Ae. albopictus, une intervention de désinsectisation du foyer en question a été effectuée, ce qui, conjointement à l'approche de la saison hivernale, a empêché de trouver d'autres exemplaires de Ae. atropalpus. En revanche, fin août 1997, neuf larves de $A e$. atropalpus ont été récoltées à l'intérieur de trois pneus usagés, pendant la surveillance entomologique menée d'avril à octobre dans le même lieu où le moustique a été récolté la première fois.

\section{MORPHOLOGIE DE AEDES ATROPALPUS}

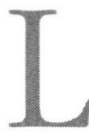

'identification des exemplaires a été effectuée sur la base des caractéristiques morphologiques proposées par O'Mehara \& Craig (1970), Zavortink (1972) et par Darsie \& Ward (1981). Ci-dessous figurent la description complète de la larve et de l'adulte, mâle et femelle d'Ae. atropalpus.

\section{LARVE}

Antenne d'une longueur d'environ la moitié de la tête, avec de rares épines; soie antennaire (1-A) bifide ou trifide, insérée à la moitié environ de la longueur de l'antenne. Soies de la tête : post-clypéale (4-C) courte et ramifiée; frontale interne (5-C) et moyenne (6-C) simples; frontale externe (7-C) avec 2-5 ramifications barbées. Soies prothoraciques (PT) : 1-PT longue, généralement double, rarement simple; 2-PT courte, simple; 3-PT courte, simple ou trifide; 4-PT courte, simple; 5-PT longue, simple ou trifide; 6-PT longue, simple, rarement bifide; 7-PT longue avec 2-4 ramifications. Soie abdominale latérale 6 normalement multifide sur les segments I-V, bifide sur le segment VI. Huitième segment abdominal avec de 21 à 58 épines. Épines du huitième segment en forme de spatule, avec marge distale denticulée et sans dent médiane plus grande. Indice siphonique compris entre 1,6 et 2; peigne muni de 12 à 24 épines, s'étendant presque jusqu'à l'extrémité apicale du siphon et dont les 2-4 dernières épines sont nettement séparées des autres; soie siphonique (1-S) insérée à l'intérieur du peigne et formée de 4 à 9 branches. Segment anal (X) recouvert presque jusqu'à la moitié par la selle, qui porte quelques épines distales; soie latérale (3-X) longue; soie caudale interne (2-X) courte et multifide; brosse ventrale (4-X) bien développée, avec toutes les touffes insérées à l'intérieur de l'aire barrée; papilles anales coniques, de 2,5 à 4 fois plus longues que la selle.

\section{FEMELLE}

Taille petite à moyenne. Tête : trompe munie d'écailles sombres; palpes courts et sombres. Occiput avec une tache médiane constituée d'écailles lancéolées, blancjaunâtre, délimité dans sa partie inférieure par une large zone d'écailles blanches et avec une tache sombre sur la partie latérale. Parties centrale de l'occiput claire; partie sub-médiane portant des sombre écailles bifides. Torus antennal de couleur marron clair à noir, généralement pourvu de quelques écailles blanches sur la surface interne. Thorax : tégument du scutum noir; scutum muni d'une large bande médiane formée de petites écailles de couleur marron-bronzé, s'élargissant postérieurement, après l'angle du scutum; scutum avec les écailles situées dans les aires antérieure, latérale et postérieure; ces écailles sont blanc-jaunâtre ou doréclair, elles forment des dessins très variables en extension. Zone pré-scutellaire bordée d'écailles doré-clair. Partie postérieure du pronotum avec des écailles étroites et sombres sur la surface dorsale et des écailles larges et claires sur la surface ventrale. Scutum avec d'étroites écailles jaunes et des soies sombres sur les lobes. Pleures avec de petites taches formées de larges écailles plates, de couleur blanc-argenté. Les écailles de la sternopleure atteignent généralement un peu plus de la moitié de l'angle antérieur et sont bien séparées de la tache située sur la zone pré-alaire. Mésépiméron glabre dans son tiers basal. Région hypostigmatale sans taches. Soies basales du mésépiméron absentes. Abdomen : tergite I avec des écailles sombres et quelques rares écailles blanches; les autres tergites sont sombres avec des bandes basales blanches. Ventre marbré avec des écailles basales blanches, ou entièrement recouvert d'écailles sombres dans sa partie apicale. Pattes : fémurs antérieurs sombres et surface postérieure claire; fémurs médians et postérieurs clairs dans la moitié basale, sombres dans la moitié distale. Tibias sombres avec extrémités blanches. Tarsomères postérieurs 1 à 4 avec un large anneau blanc aux extrémités basaux et distaux; tarsomères 5 presque entièrement blancs. Tarses antérieurs et médians de coloration similaire aux tarses postérieurs, mais avec de fins anneaux blancs sur les articles 1 et 2 et très fins sur les articles 3 et 4 ; article 5 entièrement sombre. Ailes : longueur variant de 3 à $3,5 \mathrm{~mm}$; écailles étroites et sombres, sauf celles situées à la base de la costa qui sont blanches.

\section{MALE}

Coloration analogue à celle de la femelle. Hypopygium : lobes du neuvième tergite très petits, sans 


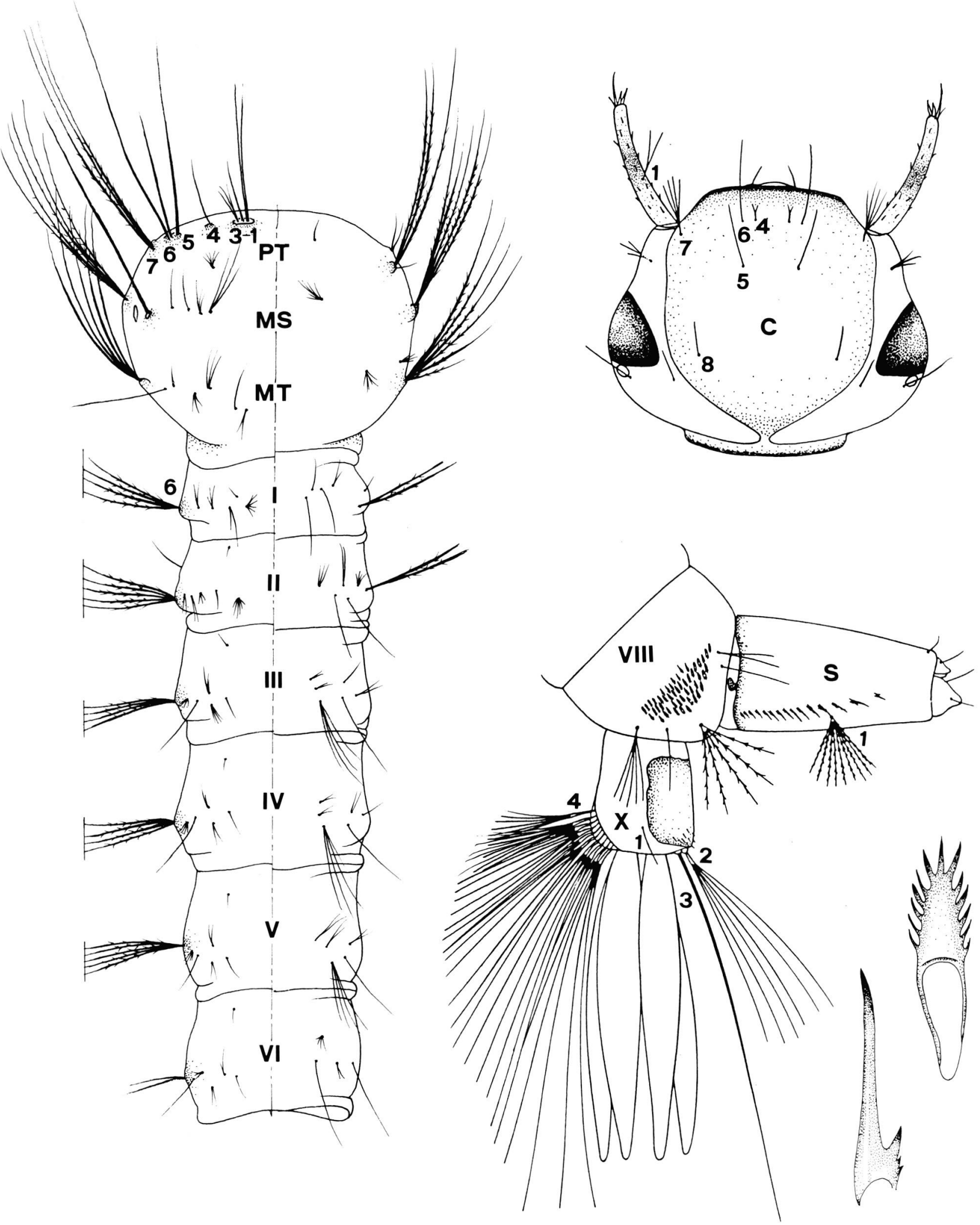

Fig. 1. - Aedes atropalpus : morphologie de la larve. 1: thorax; PT. prothorax, MS. mésothorax, MT. métathorax, et segments abdominaux I-IV. 2 : tête; C. clypeux. 3 : extrémité postérieure, VIII-X segments abdominaux, S. siphon. 4 : épine du segment VIII. 5 : épine du peigne du siphon. 

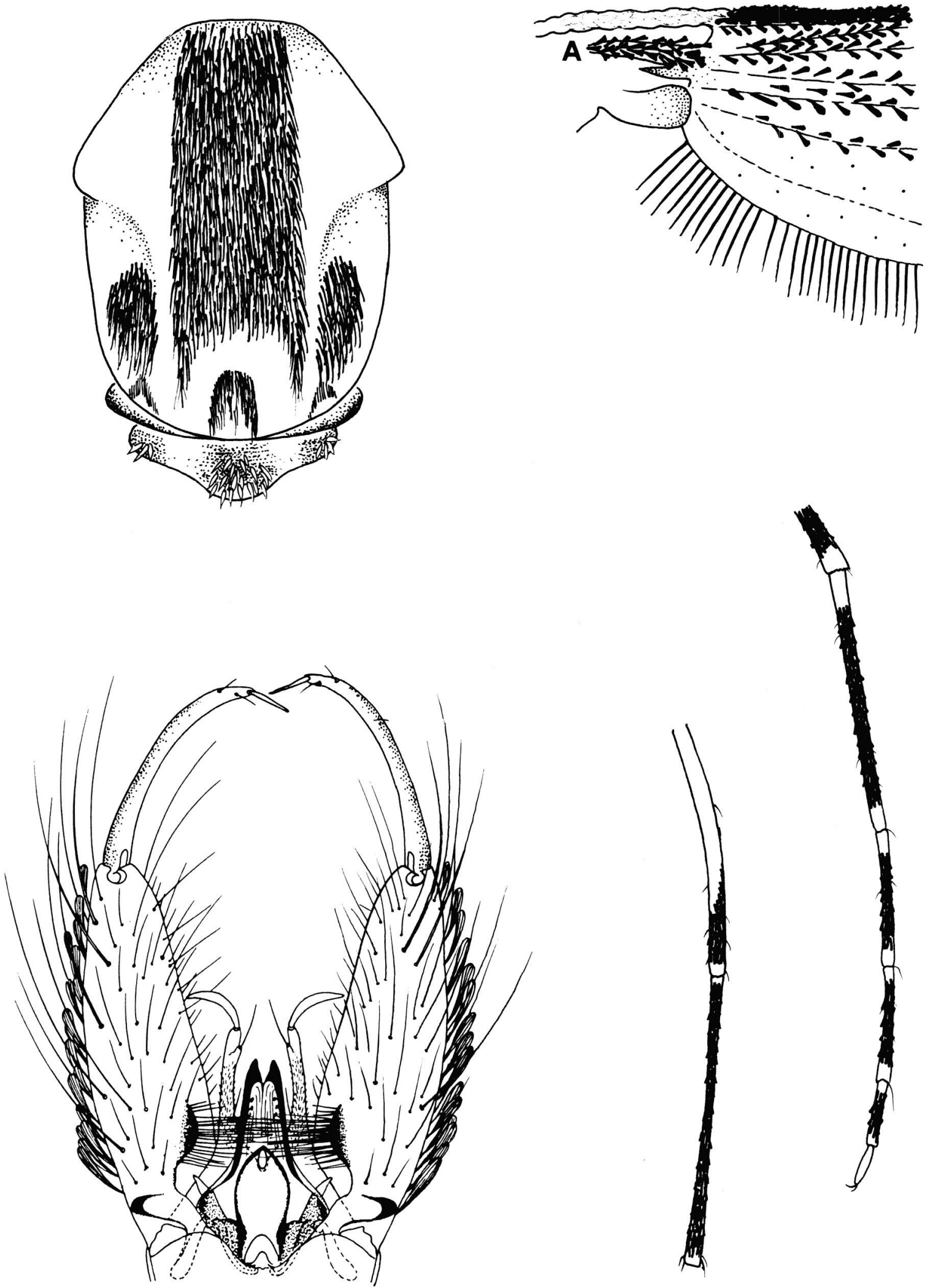

Fig. 2. - Aedes atropalpus : morphologie de l'adulte. 1 : thorax. 2 : insertion de l'aile, A. alula. $3:$ hypopygium du mâle. 4 : fémur et tibia postérieurs. 5 : tarses $1-5$ postérieurs. 
épines ni soies. Seconde moitié du dixième sternite fortement chitinisée. Édéage plus long que large, légèrement élargi à la base, arrondi à l'apex, ouvert sur sa partie ventrale. Base des claspettes étroite, d'une longueur supérieure à la troisième partie du coxite, apex poilu avec un tubercule muni d'une longue soie. Filament terminal des claspettes fin et en forme de crochet, plus court que le filament basal. Coxite d'une longueur de trois à trois fois et demie sa largeur médiane, recouvert de longues soies mêlées à des soies plus courtes et à de nombreuses écailles larges; lobe basal constitué par une petite excroissance sombre comportant une touffe de soies; lobe apical absent. Style d'une longueur égale aux deux tiers du coxite, non élargi au milieu, glabre à l'exception de deux ou trois courtes soies présentes à l'apex; épine terminale du style fine, d'une longueur égale à un septième du coxite.

Les larves de $A e$. atropalpus se distinguent facilement des 25 autres espèces de Aedes appartenant à la faune italienne par les caractères suivants :

- soies frontales internes et moyennes (5,6-C) simples; - écailles du VIII ${ }^{\mathrm{e}}$ segment abdominal en forme de spatule, sans épine médiane distincte;

- peigne du siphon avec quelques épines distales nettement séparées des autres.

Les adultes sont aussi facilement reconnaissables grâce aux caractères suivants :

- présence d'une nette tache d'écailles blanches, située

à la base de la costa, au-dessus de l'alula;

- palpes entièrement sombres;

- tarsomères postérieurs 1 à 4 avec des anneaux blancs aux extrémités basales et distales;

- tarsomères postérieurs 5 presque entièrement blancs.

\section{NOTES DE SYSTÉMATIQUE ET DE BIOLOGIE}

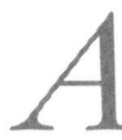

edes atropalpus est un moustique néarctique, appartenant au complexe homonyme qui comprend également : Ae. epactius Dyar \& Knab, 1908, et Ae.perichares Dyar, 1921. Les rapports systématiques entre les trois taxa sont, aujourd'hui encore, sujets à discussion (Kumm, 1940; Aitken, 1942; O'Mehara \& Craig, 1970; Munstermann, 1981; Darsie \& Ward, 1981).

Les taxa du complexe sont répandus entre, au nord le Labrador et, au sud l'Isthme de Panama (O'Mehara \& Craig, 1970). Aedes atropalpus est historiquement présent à l'ouest du Canada et aux États-Unis, à l'est du Mississippi; Ae. epactius est présent au Mexique et au sud-ouest des États-Unis (Nawrocky \& Craig 1989) et Ae. perichares en Amérique centrale (Kum et al., 1940, O'Mehara \& Craig, 1970).
Les espèces appartenant au " groupe atropalpus " sont orophiles; leurs gîtes sont constitués par les petites collections d'eau retenue dans les cavités de la roche, le long des ruisseaux, torrents et petits lacs. La découverte occasionnelle de larves d'Ae. atropalpus et d'Ae. epactius dans des gîtes créés par l'homme avait déjà été signalée en littérature depuis de nombreuses années (Shields, 1938), mais ce n'est qu'au cours des deux dernières décennies que ces deux espèces ont fréquemment été trouvées dans des récipients de diverses natures, et principalement dans des pneus usagés (O’Mehara, 1970; Munstermann, 1980; Darsie \& Ward, 1981). La colonisation des pneus usagés et le commerce de ces derniers a notablement augmenté l'aire de distribution d'Ae. atropalpus et Ae. epactius. Ainsi, dans certains états américains, l'aire de distribution la plus orientale de l'un se superpose à la plus occidentale de l'autre, engendrant ainsi des populations aux caractéristiques biologiques et biochimiques intermédiaires (Restifo \& Lanzaro, 1980; White \& White, 1980 ; Beier et al., 1983; Berry \& Craig, 1984; Andreadis, 1988; Nawrocki \& Craig, 1989).

Ae. atropalpus est une espèce multivoltine, capable de passer la saison froide à l'état d'œuf; sa diapause est liée à l'exposition du dernier stade larvaire et de pupe à de courtes photopériodes ( $<14$ heures) (O' Mehara \& Craig, 1970; Kalpage \& Brust, 1974). Comme c'est le cas pour Ae. albopictus, les femelles qui éclosent après s'être developpées dans de telles conditions pondent des œufs qui n'éclosent que lorsque les conditions de lumière et de température sont favorables à leur développement. Par contre, les oufs pondus en été sont déposés, pour la plupart, directement à la surface de l'eau (Berry \& Craig, 1984) et éclosent immédiatement. Ae. atropalpus présente un degré d'autogénie important puisqu'il est capable de pondre plus de 100 œufs lors du premier cycle gonotrophique, sans avoir effectué un repas de sang (Hudson, 1970; Bowen et al., 1994).

Ae. atropalpus semble avoir une capacité de déplacement assez réduite; il présente une activité trophique principalement diurne et pique aussi bien l'homme que les autres mammifères sauvages et domestiques (Berry \& Craig, 1984).

Le cycle de développement d'Ae. atropalpus est généralement rapide : en conditions optimales il ne dure que 5 à 9 jours (O' Mehara \& Craig, 1970); les imagos atteignent leur maturité sexuelle un jour après l'éclosion.

\section{IMPORTANCE SANITAIRE}

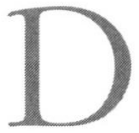

ans son habitat naturel Ae. atropalpus est considéré comme une espèce relativement peu fréquente et peu agressive pour 
l'homme; il est au plus source de nuisance au proche voisinage des gîtes. Cependant, depuis que certaines populations ont commencé à se développer dans les pneus usagés, donc dans des zones proches des agglomérations urbaines, l'espèce peut constituer un problème sanitaire. En effet ces populations peuvent prendre leur premier repas de sang et constituer ainsi une importante nuisance (Restifo \& Lanzaro, 1980; Andreadis, 1988).

Cependant, Ae. atropalpus n'est pas considéré comme un vecteur particulièrement important d'agents pathogènes. Toutefois, en laboratoire, il a été infecté avec Plasmodium gallinaceum (Trembley, 1946) et avec différents arbovirus, dont celui de l'encéphalite de La Crosse et de l'encéphalite de Saint Louis; il a ainsi transmis les virus de façon verticale, par voie transovarienne soit à travers les oeufs autogéniques, soit à travers les œufs anautogéniques (Pelz \& Freier, 1990).

\section{CONCLUSIONS}

E $\mathrm{n}$ attendant de pouvoir confirmer ou non la présence de colonies stables d'Ae. atropalpus en Italie, se repose dès maintenant le problème plus général lié à l'importation d'espèces de moustiques vecteurs potentiels d'agents pathogènes à travers le commerce des pneus usagés. Ces espèces pourraient soit s'insérer dans le cycle de la transmission des agents pathogènes locaux (dirofilaires et plasmodies aviaires), soit en introduire de nouveaux.

Comme Ae. albopictus, Ae. atropalpus pourrait trouver, sous nos latitudes, des conditions écologiques favorables à son développement et à sa diffusion. Il est donc nécessaire qu'au niveau international des mesures soient prises pour réglementer, du point de vue de la prophylaxie sanitaire, le commerce de matériels permettant le transport et la survie d'arthropodes.

Au niveau européen, l'institution d'un système de surveillance spécifique pourrait permettre l'échange, en temps réel, des informations acquises sur le terrain par les opérateurs, afin de prévenir la diffusion des espèces accidentellement introduites. Mais, c'est au niveau local que doivent être adoptées des dispositions prévoyant l'inspection et la désinfection des chargements de pneus usagés provenant de l'étranger, ainsi que leur stockage à l'abri de la pluie. En outre, il est essentiel de mettre à la disposition des opérateurs concernés des instruments d'identification performants et simples d'utilisation. Le développement de l'informatique permet de mettre au point de tels outils pour l'identification rapide et sûre des espèces appartenant aux faunes locales ou internationales (Romi et al., 1996).

\section{REMERCIEMENTS}

Nous remercions J. Brunhes pour ses conseils et pour avoir corrigé le texte en français.

\section{RÉFÉRENCES}

Aitken T.H.G. Contributions toward a knowledge of the insect fauna of Lower California. $\mathrm{N}^{\circ} 6$, Diptera Culicidae. Proceedings of the Californian Academy Sciences, 1942, 24 , $161-170$

ANDREADIS T.G. A survey of mosquitoes breeding in used tire stockpiles in Connecticut. Journal of American Mosquito Control Association, 1988, 4, 256-260.

Beier J.C., Travis M., Patricoski C. \& Kranzfelder J. Habitat segregation among larval mosquitoes (Diptera : Culicidae) in tire yards in Indiana. USA. Journal of Medical Entomo$\log$, 1983, 20, 76-80.

Berry W.J. \& Craig G.B. Jr. Bionomics of Aedes atropalpus breeding in scrap tires in northern Indiana. Mosquito News, 1984, 44, 476-484.

Bowen M.F., Davis E.E., Haggart D. \& Romo J. Host-seeking behavior in the autogenous mosquito Aedes atropalpus. Journal of Insect Physiology, 1994, 40, 511-517.

Dalla Pozza G. \& Majori G. First record of Aedes albopictus establishment in Italy. Journal of American Mosquito Control Association, 1992, 8, 318-320.

Dalla Pozza G., Romi R. \& Severini C. Source and spread of Aedes albopictus in the Vento region of Italy. Journal of American Mosquito Control Association, 1994, 10, 589-592.

DARSIE R.F. JR. \& WARD R.A. Identification and geographical distribution of the mosquitoes of north America, north of Mexico. Mosquito Syst. Suppl., 1981, 1, 1-313.

Hudson A. Factors affecting egg maturation and oviposition by autogenous Aedes atropalpus (Diptera: Culicidae). Canadian Entomology, 1970, 102, 939-949.

Kalpage K. \& Brust R.A. Studies on diapause and female fecundity in Aedes atropalpus. Environmental Entomo$\log y, 1974$, 3, 139-145.

Knudsen A.B., Romi R. \& Majori G. Occurrence and spread in Italy of Aedes albopictus with implications for its introduction into other parts of Europe. Journal of American Mosquito Control Association 1996, 12, 177-183

Kumm H.W., Komp W.H.W. \& Ruzz H. The mosquitoes of Costa Rica. American Journal of Tropical Medicine, 1940, $20,385-422$

Munstermann L.E. Distinguishing strains of the Aedes atropalpus group (Diptera: Culicidae) by analysis of enzime variation. Annals of the Entomological Society of American, 1980, 73, 699-704.

NAwrocki S.J. \& Craig G.B. Jr. Further extension of the range of the rock pool mosquito, Aedes atropalpus, via tire breeding. Journal of American Mosquito Control Association, 1989, 5, 110-114. 
O'Meara G. \& Craig G.B. Jr. Geographical variation in Aedes atropalpus (Diptera : Culicidae). Annals of the Entomological Society of America, 1970, 63, 1392-1400.

Pelz E.G. \& Freier J.E. Vertical transmission of St. Louis encephalitis virus to autogenously developed eggs of Aedes atropalpus mosquitoes. Journal of American Mosquito Control Association, 1990, 6, 658-661.

Restifo R.A. \& Lanzaro G.C. The occurrence of Aedes atropalpus (Coquillet) breeding in tires in Ohio and Indiana. Mosquito News, 1980, 40, 292-294.

Romi R. History and updating of the spread of Aedes albopictus in Italy. Parassitologia, 1995, 37, 99-103.

Romi R. Linee guida per la sorveglianza ed il controllo di Aedes albopictus in Italia. Rapporti ISTISAN, 1996, 96/4. $51 \mathrm{p}$.

Romi R., Sabatinelli G. \& Pontuale G. Computer-aided identification of the italian mossquitoes (Diptera: Culicidae). Parassitologia, 1996, 38 (1-2), 123.

Sabatini A., Raineri V., Trovato G. \& Coluzzi M. Aedes albopictus in Italia e posssibile diffusione della specie nell'area mediterrranea. Parassitologia, 1990, 32, 301-304.

Trembley H.L. Aedes atropalpus (Coq.) a new mosquito vector of Plasmodium gallinaceum Brumpt. Journal of Parasitology, 1946, 2, 499-501.

White D.J. \& White J.P. Aedes atropalpus breeding in artificial containers in Suffolk County, New York. Mosquito News, 1980, 40, 106-107.

Zavortink T. Mosquito studies XXVII. The new world species formerly placed in Aedes (Finlaya). Contributions of the American Entomological Institute, 1972, 8, 1-206.

Reçu le 24 mai 1997 Accepté le 8 juillet 1997 